\title{
Is there a role for contraceptive vaccines in fertility control?
}

\author{
A JAGANNADHA RAO \\ Department of Biochemistry and Department of Molecular Reproduction, Development and Genetics, \\ Indian Institute of Science, Bangalore 560 012, India
}

(Fax,91-80-3600814; Email,ajrao@biochem.iisc.ernet.in)

The eradication of small pox using the mass vaccination approach has been the main impetus for our efforts to develop a contraceptive vaccine. In fact, long before the idea of developing vaccines for prevention of cholera or tuberculosis or other infectious diseases was considered, efforts were in progress for the development of a contraceptive vaccine for controlling the population growth, particularly in the developing countries. The success of the use of vaccine for small pox, the ease of operation, cost effectiveness and long term protection, were some of the factors which were the driving forces in the efforts to develop contraceptive vaccines. However, almost a quarter century after the idea was first considered, investment of considerable amount of resources and efforts of several competent teams, we are no where near the actual use of the proposed vaccines. The concept of contraceptive vaccines, is radically different from conventional vaccines for infectious diseases. Unlike the vaccines for infectious diseases such as cholera, tuberculosis and small pox wherein no physiological process is interfered with, in the immunized subject, the use of contraceptive vaccine is intended for interfering with a physiological process associated with reproduction in normal healthy subjects. In addition, the antigens used are the isologous antigens from egg, sperm or certain reproductive hormones. Since these isologous antigens may not be as immunogenic as foreign antigens as in the case of antigens from organisms that are responsible for infectious diseases, maintenance of the antibody titer in the immunized subjects over a period is a problem (Herr 1996). A contraceptive vaccine essentially involves the use of a molecule, either a protein or a small ligand which has a critical role in the reproductive process. Interfering with its function by using antibodies would mean an impairment in reproduction. The primary requirement for a molecule to be a candidate vaccine is that it should have an indispensable specific role in the reproductive process and its absence should not have any adverse effects other than impairing fertility. Besides it should be easy and economically viable to produce on a large scale. Over the years several candidate vaccines have been proposed for contraception both in the male and female.

Historically, many more efforts have been directed towards development of immuno-contraceptives for use in the female because in a traditionally male-dominated society such as ours, family planning has primarily been considered as the woman's responsibility. In addition, our knowledge about the female reproductive system has preceded our understanding about the male reproductive system. Also, the sites of interception in the female reproductive systems are many more as compared to that in the male. Further, in the case of the female, one has to deal with only one egg, while in the male, the proposed method should be capable of interfering with the fertilizing ability of thousands of sperms.

Over the years, several reproductive hormones have been identified as potential targets, both in the male and female, such as gonadotropin releasing hormone $(\mathrm{GnRH})$, follicle stimulating hormone (FSH), luteimizing hormone (LH), human chorionic gonadotropin (hCG) and gonadal steroids. In addition, the antigens of trophoblasts and early embryo, egg surface antigens and sperm surface antigens have also been suggested as possible candidates. Of the reproductive hormones, one which has been proposed as an immunocontraceptive for both the male and female is $\mathrm{GnRH}$ the decapeptide produced by the hypothalamus, which regulates the synthesis and secretion of both FSH and LH, the two important pituitary hormones involved in reproduction both in males and in females. Administration of antibodies to GnRH resulted in

Keywords. Fertility control; immunocontraception 
the impairment of fertility both in the male and female animal models (Ladd et al 1990; Talwar et al 1993). However, neutralization of GnRH also resulted in the decrease in libido and functions of other accessory reproductive organs in addition to osteoporosis in females and loss of body weight in males (due to decrease in the level of gonadal steroids). These effects however minor they may be, are not acceptable. Efforts are in progress to supplement suitable steroids (androgens and estrogens) using a variety of delivery systems. However, the precise dose of the steroid hormone, which does not have side effects (on the prostate by androgen, uterus and mammary gland by estrogen) have to be arrived at and we are far from the practical application of this approach.

Yet another reproductive hormone which has shown considerable promise as a candidate vaccine is hCG which is indispensable for maintenance of early pregnancy in the human female. Both animal and preliminary studies with human females have clearly established its efficacy in interfering with pregnancy (Talwar et al 1994). However, the major problem in application of this approach has been the lack of uniform response, which is effective in interfering with pregnancy in all the immunized subjects and the necessity for use of alternative contraceptives until the required titre is obtained. In addition, due to the fear of HIV contamination, the use of hCG isolated from the urine of human subjects has not been possible and until sufficient quantities of recombinant hCG as well as methods to elicit uniform and sustained immune response in all the subjects become available, the approach is far from becoming a reality. Riboflavin carrier protein (RCP) is yet another protein which has been demonstrated to have an important role in the transport of the important vitamin riboflavin in situations wherever a blood/tissue barrier operates (e.g. blood/testis: blood/placenta etc.), has been suggested as a candidate vaccine for fertility control both in males and females (Adiga et al 1997). However, considerable work in terms of its mode of action, toxicology and clinical trials needs to be done before RCP can find its place as a candidate vaccine.

Yet another group of antigens, suggested as vaccines for immuno contraception in the female are the Zona antigens which are the egg surface specific antigens. Though animal studies have suggested the possibility of using these antigens for immunocontraception, long term studies have revealed that injection of Zona antigens results in autoimmune oophoritis indicating that immunization with Zona antigens might induce immunological attack on many eggs in the ovary resulting in premature ovarian failure (Paterson et al 1992). If this cannot be taken care of, it is unlikely that Zona antigens will have any place in immuno contraception.

In contrast to the number of targets for immunocontraception in the female, there are very few potential sites of attack in the male. One of the promising approaches, is the use of FSH as contraceptive vaccine and several studies using both rodents and monkeys as models have established that FSH is indispensable for normal spermatogenesis (Moudgal and Sairam 1998). The unique advantage of this approach is that neutralization of FSH does not have any effect on serum testosterone levels and thus does not affect libido. A phase I clinical trial has also been carried out using ovine FSH as an antigen. Considering the limited options available for immunocontraception for use in the male, the use of FSH appears to be promising as a contraceptive vaccine. However, just as in the case of hCG, constraints in the availability of sufficient quantities of recombinant $\mathrm{FSH}$, methods to sustain uniform effective response in all the immunized subjects, and the need to use alternative methods of contraception until the required titres are reached, are the major deterrents for the practical application of this method.

There have also been attempts to employ sperm surface antigens as potential candidates for immunocontraception. The objective of these efforts is to use sperm-based antigens to develop an immunocontraceptive that will produce antibodies in the female reproductive tract to block fertilization by agglutinating or immobilizing sperms (Shaha 1999).

LDH4 is an isoenzyme of lactate dehydrogenase, a glycolytic enzyme that is found only in male germ cells and detailed studies have been carried out on its structure and function. Studies carried out using baboons have revealed only partial efficacy for use as an antifertility agent (O'Hearn 1995). Other antigens which are being tested in experimental animals, include RSA-1 human SP17, Tcle-1, $\beta$ H20, SP10 (Harrison and Rosenfield 1996). However, none of the studies have reached a stage where it can be inferred that a particular antigen can be the candidate for use as a contraceptive vaccines.

It is very essential to realize that even after a candidate antigen is identified and produced in enough quantities by recombinant methods and methods are developed to sustain an effective uniform response in $100 \%$ of the subjects (which is a daunting task), the main drawbacks in the application of immunocontraception is the need for periodic injections (which will be a major practical problem in a country like ours with a huge rural population) and also need for alternate contraceptive approaches required until sufficient titres are obtained in the subjects. This is all the more so in developing an immuno-contraceptives for use in males, since theoretically, even one sperm is adequate for fertilization and an absolute need for $100 \%$ efficacy is required. Currently efforts are in progress to employ epididymal antigens since they are believed to be involved in the process of maturation of sperms without which, under normal 
conditions sperms are incapable of fertilizing the egg (Sriraman and Rao 2001). Thus, it is hoped that by interfering with the process of sperm maturation in the epididymis, an ideal contraceptive can be developed without interfering with the libido. However, even if this is achieved, the main problem is the delivery of the specific antibody to the epididymis to interfere with the process of sperm maturation. One way to overcome the problems of lack of uniform response, periodic boosters and need for alternative methods until sufficient titre is reached, at least in the females is to use high affinity, bio-neutralizing humanized orally active recombinant antibodies which can be ingested at the appropriate period in the cycle. Although this sounds unrealistic, this approach can become a reality with the present day technology.

Considering all the facts mentioned above, one might conclude that immunocontraception has no place in fertility control. To quote from a report by the committee on contraceptive research and development: "There has been much discussion of the desirability of curtailing investment in immunocontraception, primarily in response to concerns expressed by some women's groups and by individual analysts, that immunocontraception will not prove to be a biomedically appropriate contraceptive option for women in general and that research and development investment in the area would be better placed elsewhere". However, one should realize that immunocontraception is an important tool in identifying the potential target and the candidate antigens. The approach has the power to provide insights into fundamental immunological and physiological structure and mechanism that could enrich development of the applications. Following the identification of the candidate molecule using the immunological approach, it is the modern approach of understanding the molecular basis of interaction of the hormone with it receptor, which will provide the basis for developing non-protein/non-peptide based orally active mimetics to interfere with the action of the hormone. Thus, the knowledge gained out of the immunological approach will be indispensable in developing future contraceptives although immunocontraception itself may not have a place of its own in fertility control programme at the moment.

\section{References}

Adiga P R, Subramanian S, Rao J and Kumar M 1997 Prospects of riboflavin carrier protein (RCP) as an antifertility vaccine in male and female mammals; in Human reproduction update (ed.) R G Edward, vol. 3, pp 325-334

Harrison Polly F and Rosenfield Allan (eds) 1996 Contraceptive technology and the state of the science: New horizons (Washington DC: National Academy Press) pp 125165

Harrison Polly F and Rosenfield Allan (eds) 1996 Contraceptive research and development (Washington DC: National Academy Press) p. 12

Ladd A, Tsong Y Y, Lok J, et al 1990 Active immunization against LHRH I: Effects of conjugation site and dose; Am. J. Reprod. Immunol. 22-56

Herr J C 1996 Immunocontraceptive approaches; in Contraceptive research and development (eds) Polly $\mathrm{F}$ Harrison and Allan Rosenfield (Washington DC: National Academy Press) pp 401-429

Moudgal N R and Sairam M R 1998 Is there a true requirement for FSH in promoting spermatogenesis and fertility in primates?; Hum. Reprod. 113 916-919

O'Hearn P, Bambra C, Isahakia M and Goldberg E 1995 Reversible contraception in female baboons immunized with a synthetic epitope of sperm specific LDH; Biol. Reprod. 52 331-339

Paterson M, Koothan T, Morris K, O'Byrne K T, Braude P, Williams A and Aitken R J 1992 Analysis of the contraceptive potential of antibodies against native and deglycosylated porcine $\mathrm{ZP}_{3}$ in vivo and in vitro; Biol. Reprod. 46 $523-534$

Shaha C 1999 The sperm vaccine - an utopain goal?; in Male contraception: Present and future (eds) M Rajalakshmi and P D Griffin (New Delhi: New Age International Ltd) pp 319328

Sriraman V and Rao A J 2001 Current status in development of male contraceptives; in Current status in fertility regulation: indigenous and modern Approaches (eds) S R Chowdhury, C M Gupta and V P Kamboj (Lucknow: Army Printing Press) pp. 163-178

Talwar G P, Singh O, Pal R, Chatterjee N, Arunan K, Sad S, Raghunathan V, Sahai P, Gupta H and Salunke D 1993 Vaccine against LHRH and hCG; in Immunology of reproduction (ed.) R Naz (Boca Raton: CRC Press) pp 235249

Talwar G P, Singh O, Pal R, Chatterjee N, Sahai P, Dhall K, Jasvinder K, Das S K, Suri S, Buckshee K, Saraya L and Saxena B L 1994 A vaccine that prevents pregnancy in women; Proc. Natl. Acad. Sci. USA $918532-$ 8536 